\begin{tabular}{|c|c|}
\hline Title & Role of anion ordering and effective pressure in the field-induced spin-density-wave phase of (TMTSF)(2)X \\
\hline Author(s) & Minamidate, T.; Matsunaga, N.; Nomura, K.; Sasaki, T. \\
\hline Citation & $\begin{array}{l}\text { EPL, 115(3), } 37002 \\
\text { https://doi.org/10.1209/0295-5075/115/37002 }\end{array}$ \\
\hline Issue Date & $2016-08$ \\
\hline Doc URL & http:/hdl .handle.net/2115/66935 \\
\hline Type & article (author version) \\
\hline File Information & 20161115_TMTSF2Re04_FISDW_HUSCUP.pdf \\
\hline
\end{tabular}

Instructions for use 


\title{
Role of anion ordering and effective pressure in the field-induced spin-density-wave phase of (TMTSF) ${ }_{2} X$
}

\author{
T. Minamidate ${ }^{1}$, N. Matsunaga ${ }^{1}$, K. Nomura ${ }^{1}$ and T. Sasaki ${ }^{2}$ \\ 1 Department of Physics, Hokkaido University - Sapporo 060-0810, Japan \\ 2 IMR, Tohoku University - Sendai 980-8577, Japan
}
PACS 75.30.Fv - Magnetic properties and materials; Spin-density waves
PACS 75.47.-m-Magnetic properties and materials; Magnetotransport phenomena; materials for magnetotransport
PACS 74.70.Kn - Superconductivity; Organic superconductors

\begin{abstract}
Magnetoresistance and Hall resistance measurements were conducted in the fieldinduced spin-density-wave (FISDW) phase of (TMTSF) ${ }_{2} \mathrm{ReO}_{4}$ above $1.0 \mathrm{GPa}$, with an anion ordering specified by $Q_{\mathrm{AO}}=(0,1 / 2,1 / 2)$. The quantized Hall resistance shows the sequence $N=$ $0,1,2,-2,4,-4,-6,-8, \cdots$, with decreasing field that is successfully explained by the "extended standard model". Consequently, we demonstrate that the difference between the chemical and hydrostatic pressures is linked to the appearance of the peculiar FISDW phase of the TMTSF salts with $Q_{\mathrm{AO}}=(0,1 / 2, *)$.
\end{abstract}

Introduction. - Quasi-one-dimensional (Q1D) organic conductors (TMTSF $)_{2} X$, in which TMTSF denotes tetramethyltetraselenafulvalene and $X$ is a group such as $\mathrm{PF}_{6}, \mathrm{ClO}_{4}$ or $\mathrm{ReO}_{4}$ have been extensively investigated because of their rich phase diagrams due to the presence of superconducting, spin-density-wave (SDW) and field-induced SDW (FISDW) phases [1]. The FISDW phase diagram in the $\mathrm{PF}_{6}$ salt is classified by the quantized Hall resistance $\rho_{x y} \sim h /\left(N 2 e^{2}\right)$ and shows the sequence $N=0,1,2,3,4,5, \cdots$ with decreasing magnetic field. This phase sequence has been successfully explained using the mean field theory approach known as the "standard model" that is based on the nesting of the Q1D Fermi surface (FS). In this model, the quantum number $N$ is the number of the fully occupied Landau levels in the hole or electron pockets at the FS. However, the sequence for the FISDW phase in the relaxed $\mathrm{ClO}_{4}$ salt is given by $N=0,1,3,-1,5, \cdots$, which shows the suppression of even numbered phases. Currently, the absence of the evennumbered phases is believed to be caused by the anion ordering $(\mathrm{AO})$ with a wave vector $Q_{\mathrm{AO}}=(0,1 / 2,0)$ that separates the original FS into two FS sheet pairs [2]. The observed FISDW phase in the relaxed $\mathrm{ClO}_{4}$ salt is known to exhibit significant disagreements with the "standard model" that considered the effect of AO. This approach predicts the existence of periodic oscillation of the FISDW transition temperature $T_{\text {FISDW }}$ as a function of field $[3,4]$.
However $T_{\text {FISDW }}$ of about $5.5 \mathrm{~K}$ is essentially independent of the field above $15 \mathrm{~T}[5]$.

The negative Hall resistance expressed by a negative quantum number was reported in certain magnetic field regions of the FISDW phase. In the $\mathrm{PF}_{6}$ salt, the negative Hall plateau with an even negative quantum number was reported in the vicinity of the critical pressure [6]. Theoretically, the negative-even-number state can be explained by a model including higher order harmonic terms [7] or the umklapp scattering [8]. With the appropriately chosen parameters, both models can produce either a monotonic FISDW phase sequence or an alternating sequence of positive-integer and negative-even-number states. In the relaxed $\mathrm{ClO}_{4}$ salt, a negative Hall plateau (Ribault phase) was reported [9]. Although the Ribault phase shows strong temperature and cooling rate dependences, it is assigned $N=-1$ state based on the results shown in Fig.2 in ref. [10]. While the Ribault phase has been extensively investigated experimentally and theoretically, its origin is still an open question.

The $\mathrm{ReO}_{4}$ salt is the key material to investigate the role of $\mathrm{AO}$ in the TMTSF salts. At ambient pressure, the $\mathrm{ReO}_{4}$ salt shows the metal-insulator (MI) transition with the $\mathrm{AO}$ transition of $Q_{\mathrm{AO}}=(1 / 2,1 / 2,1 / 2)$ at $180 \mathrm{~K}$ $[11,12]$. Above $0.8 \mathrm{GPa}$, the MI transition is suppressed and the AO transition of $Q_{\mathrm{AO}}=(0,1 / 2,1 / 2)$ is induced $[11,12]$. The $k_{x}$ and $k_{y}$ components of the AO wave vector 
in the $\mathrm{ReO}_{4}$ salt above $0.8 \mathrm{GPa}$ are the same as that of the $\mathrm{ClO}_{4}$ salt. As the FS geometry is determined by the $\mathrm{AO}$ wave vector, both salts have the same geometry: two pairs of FS sheets [13]. In this pressure region of the $\mathrm{ReO}_{4}$ salt, the FISDW phase is observed with quantum numbers $N=0,1,2, \cdots$ above $16 \mathrm{~T}$ [14]. Either $\mathrm{ClO}_{4}[15,16]$ and $\mathrm{ReO}_{4}[17,18]$ salts in the metallic phase show quantum magnetic oscillation called "rapid oscillation" (RO), wherein the mechanisms are explained by the Stark quantum interference [19] and not the Shubnikov-de Haas effect. The existence of RO in the metallic phases of both salts suggests that the both salts have the same FS geometry. To elucidate the role of AO in the FISDW phase, we investigated magnetoresistance (MR) and Hall resistance in the $\mathrm{ReO}_{4}$ salt above 1.0 GPa and discussed the FISDW subphase structure. The determination of the $\mathrm{ReO}_{4}$ quantum numbers is essential for elucidating the $\mathrm{AO}$ role in the FISDW phase of the TMTSF salts.

Experimental. - Single crystals of the $\mathrm{ReO}_{4}$ salt were synthesized by the standard electrochemical method. Electric leads comprising $10 \mu \mathrm{m}$ gold wire were attached with silver paint onto the gold evaporated contacts. The size of the sample was $3.29 \times 0.64 \times 0.37 \mathrm{~mm}^{3}$. To obtain a uniform current, the current contacts covered the entire surface areas of both ends of the sample. The sample was mounted inside a beryllium-copper and $\mathrm{NiCrAl}$ hybrid clamp cell with Daphne 7373 oil as a pressure medium. The Hall resistance and MR measurements were performed using a standard six-probe dc method. The current flow direction corresponded to the $a$-axis and the Hall voltage was measured nearly along the $b^{\prime}$-axis.

Results and discussion. - Figure 1(a) shows the Hall resistance and MR data obtained in the $\mathrm{ReO}_{4}$ salt under the pressure of $1.1 \mathrm{GPa}$. In the metallic phase, we observed RO same as reported in previous paper [17,18], although it is hardly visible due to the logarithmic scale of the resistance axis. Four transitions are observed with increasing field above the initial FISDW transition at $6.5 \mathrm{~T}$ at $0.24 \mathrm{~K}$. Within the FISDW subphases, the Hall resistance is almost constant with the field. At least one positive Hall phase and three negative Hall phases are identified. The transitions between the positive Hall phase and the negative Hall phase at $10.8 \mathrm{~T}$ and $12 \mathrm{~T}$ give sharp peaks in the MR data, which suggests the high purity of our sample. Negative Hall phases appear in a very wide field range. The Hall resistance ratios of the Hall plateaus in the $12-16 \mathrm{~T}, 10.8-12 \mathrm{~T}$, and $8.2-10.8 \mathrm{~T}$ ranges are $1:-1 / 2: 1 / 2$. From the Hall resistance values [20], we assigned the Hall plateaus in the $12-16 \mathrm{~T}, 10.8-12 \mathrm{~T}$, and $8.2-10.8 \mathrm{~T}$ ranges as $\mathrm{N}==-2,4$, and -4 , respectively. No positive Hall phases were observed below 10.8 T. Figure 1(b) shows the Hall resistance below $8 \mathrm{~T}$ at a slightly smaller pressure of $1.0 \mathrm{GPa}$. Based on the temperature dependence of the Hall resistance, the phase in the $6.0-7.6 \mathrm{~T}$ range is assigned as the $N=-6$ phase. The phase in the $5.0-6.0 \mathrm{~T}$ range exhibits a negative Hall
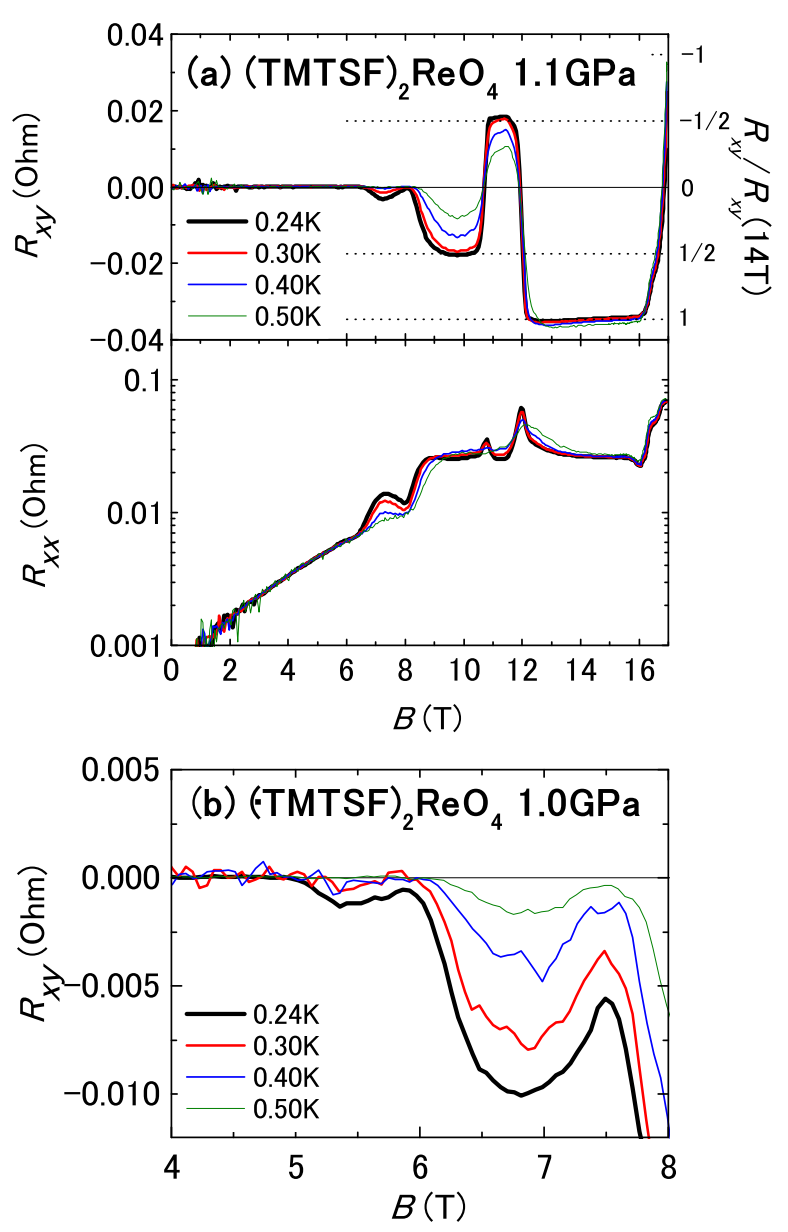

Fig. 1: (a)Hall resistance $\left(R_{x y}\right)$ and magnetoresistance $\left(R_{x x}\right)$ in the $\mathrm{ReO}_{4}$ salt for various temperature below $0.5 \mathrm{~K}$ with the field parallel to the $c^{*}$-axis at $1.1 \mathrm{GPa}$. Dotted lines are guides to recognize that the ratios correspond to the each Hall plateau. (b)Hall resistance $\left(R_{x y}\right)$ in the $\mathrm{ReO}_{4}$ salt at $1.0 \mathrm{GPa}$ below $8 \mathrm{~T}$.

resistance and is assigned as $N=-8$ phase as explained below. Thus, the resulting sequence of quantum numbers with decreasing magnetic field below $17 \mathrm{~T}$ is as follows: $N=-2,4,-4,-6,-8, \cdots$.

Figure 2 shows the FISDW phase diagram under 1.0, 1.1, and 1.2 GPa. The transition temperatures determined from the Hall resistance are denoted by open circles. At the phase boundaries between the $N=-4$ and $N=-6$ and between the $N=-6$ and $N=-8$ phases, the magnetic field dependence of $T_{\text {FISDW }}$ displays a dip. The transition temperatures for the phases with $N=4$ and $N=-4$ are almost identical. The $N=-8$ phase cannot be observed at $0.24 \mathrm{~K}$ above $1.1 \mathrm{GPa}$. With increasing pressure, the boundaries between FISDW subphases shift to higher field values, the negative Hall phases region in the phase diagram becomes narrower, and that of the $N=4$ phase becomes wider. Our results are consistent with the previous report [14] that assigned the quantum number sequence in the FISDW phase of the 


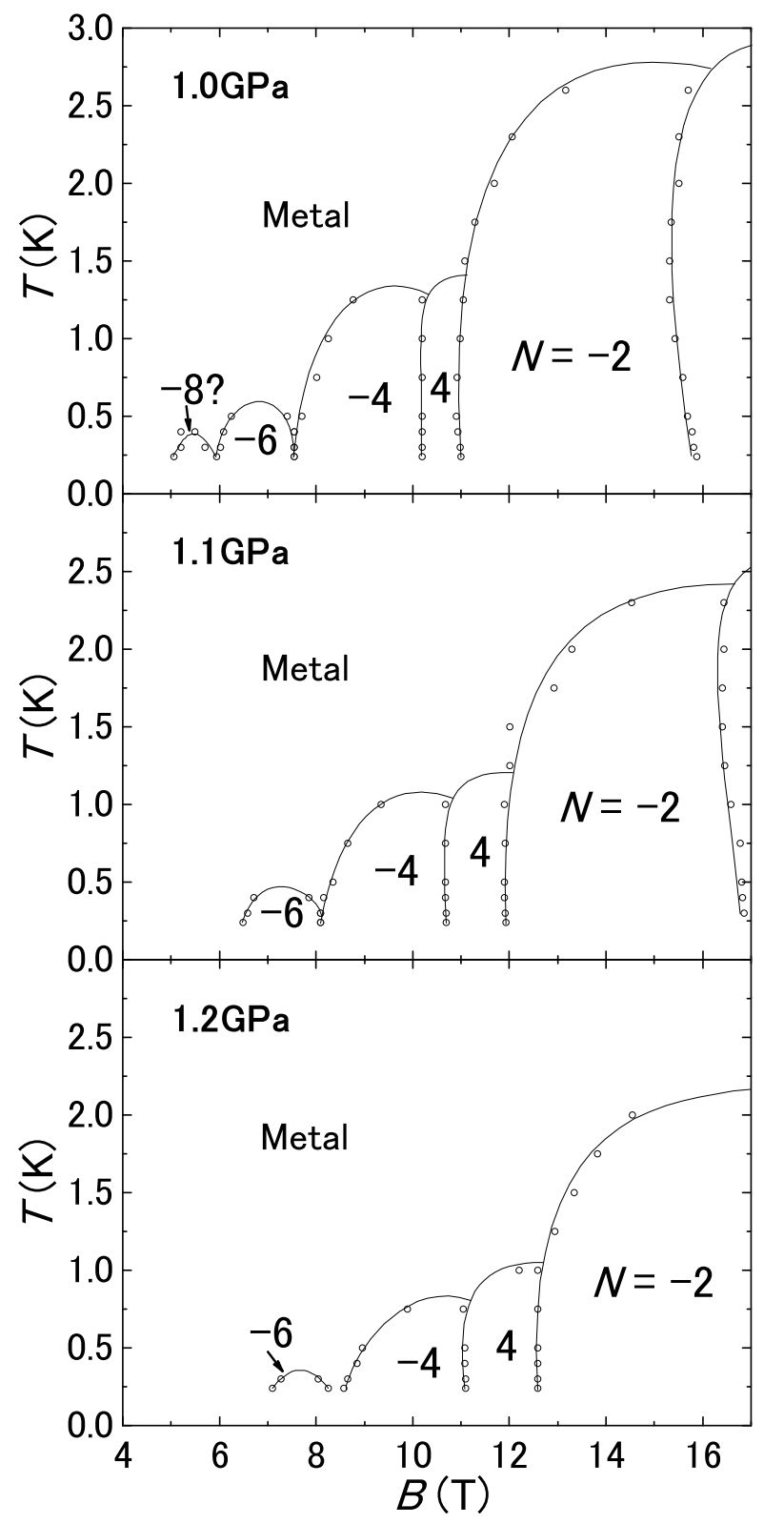

Fig. 2: Magnetic field - temperature phase diagram of the $\mathrm{ReO}_{4}$ salt under 1.0,1.1 and 1.2 GPa. The data shown by open circles is obtained from the Hall resistance measurements.

$\mathrm{ReO}_{4}$ salt as $N=0,1,2$, negative, $\cdots$ above $16 \mathrm{~T}$ at $0.5 \mathrm{~K}$. Combined with the previous report, we obtain $N=0,1,2,-2,4,-4,-6,-8$, with decreasing magnetic field as the full quantum number sequence for the $\mathrm{ReO}_{4}$ salt. Thus, the quantum number series of positive integer and negative even numbers.

The $\mathrm{ReO}_{4}$ phase diagram described above is similar to the FISDW phase diagram of the $\mathrm{PF}_{6}$ salt. the FISDW phase of the $\mathrm{PF}_{6}$ salt shows a quantum number sequence $N=0,1,2,-2,3,4,5,-4, \cdots$ under $0.65 \mathrm{GPa}$, which is the critical pressure for suppressing the SDW phase at $0 \mathrm{~T}$ [6]. Moreover, the structure of the FISDW phase in the $\mathrm{PF}_{6}$ salt can be described by the competition be-
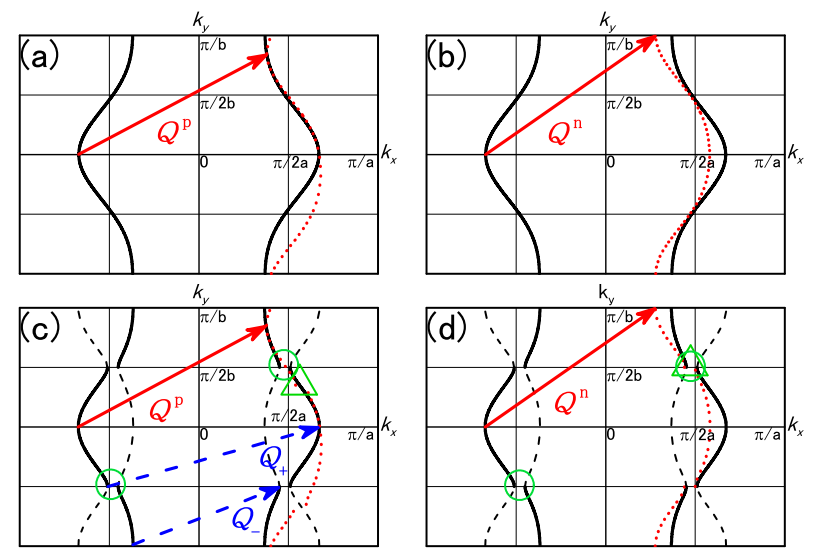

Fig. 3: Black bold lines denote FS. Red dotted lines denote the left side of FS nested by the nesting vector. Bold and dashed lines in (c) and (d) denote FS constructed by the AO, which are written by the periodic zone scheme. (a)Nesting vector $Q^{\mathrm{p}}=\left(2 k_{\mathrm{F}}+N G, \pi / b+\delta Q_{\perp}\right)$ in the system with one FS pair. One hole pocket is generated in the first Brillouin zone using the periodic potential with $Q^{\mathrm{p}}$. (b)Nesting vector $Q^{\mathrm{n}}=$ $\left(2 k_{\mathrm{F}}+N G, \pi / b\right)$ in the system with one FS pair. Two electron pockets are created by $Q^{\mathrm{n}}$. (c)Red arrow with bold line: $Q^{\mathrm{p}}$ in the system with two FS pairs divided by AO gap. Blue arrows with broken line: nesting vectors $Q_{ \pm} \cdot Q_{+(-)}$denotes the nesting between outer (inner) FS. (d)Nesting vector $Q^{\mathrm{n}}$ in the system with two FS pairs.

tween the positive integer Hall phase and the negative even Hall phases. With increasing pressure, the negative Hall phases are gradually suppressed, disappearing completely for pressures above 1.0 GPa and leaving the sequence of the positive Hall phases specified by $N=0,1,2,3,4, \cdots$ [21]. Thus, except for the wider negative $N$ phase of the $\mathrm{ReO}_{4}$ salt, the FISDW phases in the $\mathrm{ReO}_{4}$ and $\mathrm{PF}_{6}$ salts show similar quantum number sequences and similar pressure dependence.

We now discuss the physical origin of the FISDW phase diagrams in these systems. In the simple "standard model", the FISDW state is stabilized by the nesting vector shown in Fig.3(a) and given by $Q^{\mathrm{p}}=$ $\left(2 k_{\mathrm{F}}+N G, \pi / b-\delta Q_{\perp}\right)$, where $k_{\mathrm{F}}$ is Fermi wave number; $G=e b B / \hbar ; b$ is the $b$-axis lattice parameter; $B$ is the magnetic field; and $\delta Q_{\perp}$ is the deviation from $\pi / b$ along the $k_{y}$ direction. Using the nesting vector $Q^{\mathrm{p}}$ generates one hole pocket in the first Brillouin zone in the entire range of magnetic field strengths. Therefore, in the framework of the simple "standard model", only the positive integer states are stabilized by the hole Landau orbitals. To explain the presence of negative even- $N$ states, Zanchi et al. introduced higher harmonics terms [7], whereas Dupuis et al. introduced the umklapp process [8] into the energy dispersion. Hereafter, we will refer to these models as the "extended standard model". In the "extended standard model", even- $N$ phases appear for $Q^{\mathrm{n}}=\left(2 k_{\mathrm{F}}-N G, \pi / b\right)$ (Fig.3 (b)). In the case of $Q^{\mathrm{n}}$, the FISDW state with two quantized electron (hole) pockets of equal size is sta- 
bilized for negative (positive) $N$. These FISDW phases are the quantized Hall states with an even number. If the effects of the third order harmonic terms [7] or the umklapp scattering [8] are considered, FISDW phases with $Q$, in which $k_{y}$ does not equal to $\pi / b$, are suppressed. Furthermore, when we consider the fourth harmonic term, the sign of which is equal to that of the second harmonic term, a phase with electron pockets is favored. According to these theories, the FISDW phase can be described by the competition between the positive integer states and the negative even number states; therefore, the quantum number series observed in the $\mathrm{ReO}_{4}$ salt can be explained by the "extended standard model" in the same manner as the phases observed for the $\mathrm{PF}_{6}$ salt.

To explain the wide stability range of negative-even- $N$ phases in the $\mathrm{ReO}_{4}$ salt, we now consider the effect of the energy gap because of AO within the "extended standard model". In the rectangular lattice model with the superlattice because of $\mathrm{AO}$ along the $b$ direction, the FS is split into two pairs of slightly warped parallel sheets. In Fig. 3 (c)(d), the black bold lines denote the FS plotted by the extended zone scheme and the bold and dashed lines denote the FS plotted by the periodic zone scheme. The positive integer $N$ phases correspond to $Q^{\mathrm{p}}$ with $k_{y}=\pi / b+\delta Q_{\perp}$ (Fig.3(c)). In this case, $\mathrm{AO}$ gaps open at both $k_{y}=\pi / 2 b$ on the original FS (open circles in Fig.3(c)) and $k_{y}=\pi / 2 b+\delta Q_{\perp}$ on the shifted FS (open triangles in Fig.3(c)). Therefore, the nesting with $Q^{\mathrm{p}}$ becomes imperfect because of the AO gap. However, in the case of the negative-even- $N$ phases corresponding to $Q^{\mathrm{n}}$ with $k_{y}=\pi / b($ Fig. $3(\mathrm{~d}))$, the nesting does not become imperfect because both gaps of nested FS open at the same position, i.e., $k_{y}=\pi / b$. Therefore, the negative-even- $N$ phases survive in the presence of a large AO gap. Accordingly, we can assign the negative Hall phase in the 5.0 $6.0 \mathrm{~T}$ range at $1.0 \mathrm{GPa}$ to $N=-8$.

Next, we compare the FISDW phase in the $\mathrm{ReO}_{4}$ salt with those of the $\mathrm{ClO}_{4}$ salt. Both salts exhibit an AO gap because of the superlattice potential along the $b$-axis. In both salts, RO in MR was observed in metallic phase. The origin of $\mathrm{RO}$ in the metallic phase is thought to be the Stark quantum interference effect [19]. The Stark effect occurs in the case, in which an electron proceed along two alternative trajectories plotted by bold and dashed lines in Fig. 3(c)(d). The size of the AO gap $\left(E_{\mathrm{g}}\right)$ in the $\mathrm{ClO}_{4}$ salt has been estimated as $4.5 \mathrm{meV}$ based on the magnetic field dependence of the RO in MR by assuming the Fermi energy $E_{\mathrm{F}}$ of $0.1 \mathrm{eV}$ using the Stark model [16]. Because this $E_{\mathrm{F}}$ is much smaller than the value expected from tight binding calculations, we used $E_{\mathrm{F}}=0.4 \mathrm{eV}$ in our analysis. Using $E_{\mathrm{F}}=0.4 \mathrm{eV}$, Komada et al. have obtained $E_{\mathrm{g}} \approx 10$ meV for the $\mathrm{ClO}_{4}$ salt and $E_{\mathrm{g}} \approx 12 \mathrm{meV}$ for the $\mathrm{ReO}_{4}$ salt [22]. Therefore, we consider the AO gap for the $\mathrm{ReO}_{4}$ salt is almost the same as or slightly larger than that of the $\mathrm{ClO}_{4}$ salt. In the $\mathrm{ClO}_{4}$ salt at ambient pressure, the $N$ sequence is reported as $N=0,1,3,-1,5, \cdots[9]$, indicating the suppression of the even- $N$ phases. While it has been believed that this suppression can be explained by the "standard model" taking into account the AO gap [4], the predicted oscillation of $T_{\text {FISDW }}$ as a function of field was not experimentally observed in the $\mathrm{ClO}_{4}$ salt. However, the FISDW phase in the $\mathrm{ReO}_{4}$ salt does not show the suppression of the even- $N$ phases despite the larger $\mathrm{AO}$ gap. Therefore, the "standard model" with AO cannot completely explain the FISDW phase diagram of the $\mathrm{ClO}_{4}$ salt.

In our previous study, based on the cooling rate dependence of the FISDW phase, we reported that the FISDW in the $\mathrm{ClO}_{4}$ salt is stabilized with the nesting vector $Q_{+(-)}$, which denotes the nesting between the outer (inner) FS (blue arrows with broken line in Fig.3(c)) $[5,23,24]$. The $k_{y}$ component of $Q_{+(-)}$is $\pi / 2 b$, which is approximately half of the $Q^{\mathrm{p}}$ value. Theoretically, a large AO gap stabilizes the $Q_{ \pm}$state [25]; however, we found that the FISDW phase in the $\mathrm{ReO}_{4}$ salt under the 1.0 GPa pressure can be explained within the framework of "extended standard model" with the $Q^{\mathrm{p}, \mathrm{n}}$ despite the larger AO gap. This result suggests that the stabilization of $Q_{ \pm}$state is not determined only by the size of the AO gap.

We observed that a realistic lattice model is required to accurately reproduce the experimentally observed FISDW phase sequence. Previously, FISDW phase models have assumed a simple rectangular lattice with only nearest neighbor transfer integral. In the vicinity of $E_{\mathrm{F}}$, the energy band is described by

$$
\epsilon(\mathbf{k})=\hbar v_{\mathrm{F}}\left(\left|k_{x}\right|-k_{\mathrm{F}}\right)-2 t_{b} \cos \left(b k_{y}\right)+\epsilon_{0} \cos \left(2 b k_{y}\right)
$$

where $v_{\mathrm{F}}=2 a t_{a} \sin \left(a k_{\mathrm{F}}\right)$ is the Fermi velocity; $t_{a}$ and $t_{b}$ are the electron transfer integrals; and $\epsilon_{0}=$ $t_{b}^{2} \cos \left(a k_{\mathrm{F}}\right) / 2 t_{a} \sin ^{2}\left(a k_{\mathrm{F}}\right)$ is the second harmonic term coefficient. In this model, FISDW stability is determined by both $\epsilon_{0}$ and the AO gap. Because these two parameters are essentially identical in both the $\mathrm{ReO}_{4}$ and $\mathrm{ClO}_{4}$ salts, the differences in the FISDW phases of these salts cannot be explained by the model using the simplified rectangular lattice.

The simplified model is deficient because of the triclinic form of the lattice in the $\mathrm{ReO}_{4}$ and $\mathrm{ClO}_{4}$ salts; moreover, the multi-transfer integrals in the $a-b$ plane plays a significant role as reported by Yamaji et al. [26]. Table 1 lists the lattice parameters [27] and the transfer energies calculated using the two-dimensional tight-binding model in the $\mathrm{PF}_{6}$ salt at ambient pressure and $0.7 \mathrm{GPa}$ and in the $\mathrm{ClO}_{4}$ salt at ambient pressure. The inset in Table 1 shows the assignment of the transfer integrals in the TMTSF salts: $t_{\mathrm{s} 1}$ $t_{\mathrm{s} 2}$ specify the intra-column transfer integrals, whereas $t_{\mathrm{I} 1}$, $t_{\mathrm{I} 2}, t_{\mathrm{I} 3}$ and $t_{\mathrm{I} 4}$ are the inter-column transfer integrals. The lattice parameters $a$ and $b$ in the $\mathrm{PF}_{6}$ salt are compressed by hydrostatic pressure. However, the lattice parameters in the $\mathrm{ClO}_{4}$ salt at ambient pressure are identical to those of the $\mathrm{PF}_{6}$ salt at ambient pressure, although chemical pressure because of the replacement of the anion is expected. The effect of the chemical pressure appears in the 
Table 1: Lattice parameters and transfer energies in the $a-b$ plane for the $\mathrm{PF}_{6}$ salt at ambient pressure and 0.7 GPa and for the $\mathrm{ClO}_{4}$ salt at ambient pressure at low temperature $[26,27] . \epsilon_{0}, \tau_{\cos }, \tau_{\sin }$ and $2 t_{b}^{\prime}=\left|2 \tau_{\cos }-\epsilon_{0}\right|$ were calculated by Yamaji et al [26]. Lattice parameters are given in $\AA$, lattice angles in degrees, and all energies are in meV. Inset is the arrangement of the typical TMTSF salts viewed along the long axes of the molecules and assignment of the transfer integrals.

\begin{tabular}{|c|c|c|c|c|c|c|c|c|c|c|}
\hline$X(T, P)$ & $a$ & $b$ & $c$ & $\alpha$ & $\beta$ & $\gamma$ & & & & \\
\hline $\mathrm{PF}_{6}(4 \mathrm{~K}, \mathrm{~A} . \mathrm{P})$. & 7.076 & 7.632 & 13.322 & 84.14 & 88.05 & 70.13 & & & & \\
\hline $\mathrm{PF}_{6}(1.7 \mathrm{~K}, 0.7 \mathrm{GPa})$ & 6.980 & 7.581 & 13.264 & 84.17 & 88.04 & 70.30 & & & & \\
\hline $\mathrm{ClO}_{4}(7 \mathrm{~K}$, A.P. $)$ & 7.068 & 7.638 & 13.123 & 84.50 & 88.00 & 68.92 & & & & \\
\hline$X(T, P)$ & $t_{\mathrm{S} 1}$ & $t_{\mathrm{S} 2}$ & $t_{\mathrm{I} 1}$ & $t_{\mathrm{I} 2}$ & $t_{\mathrm{I} 3}$ & $t_{\mathrm{I} 4}$ & $\epsilon_{0}$ & $\tau_{\mathrm{cos}}$ & $\tau_{\sin }$ & $2 t_{b}^{\prime}$ \\
\hline $\mathrm{PF}_{6}(4 \mathrm{~K}, \mathrm{~A} . \mathrm{P})$. & 280 & 254 & -17.8 & -47.9 & 46.9 & 5.6 & 2.31 & 2.13 & -0.98 & 1.95 \\
\hline $\mathrm{PF}_{6}(1.7 \mathrm{~K}, 0.7 \mathrm{GPa})$ & 290 & 272 & -20.6 & -53.5 & 49.8 & 6.2 & 2.41 & 2.47 & -0.92 & 2.53 \\
\hline $\mathrm{ClO}_{4}(7 \mathrm{~K}$, A.P. $)$ & 287 & 266 & -34.0 & -64.1 & 46.2 & 7.5 & 2.23 & 3.20 & 0.55 & 4.17 \\
\hline
\end{tabular}

lattice angle $\gamma$. In the TMTSF salts, $\pi$-molecular orbitals are formed primarily from the $p_{z}$-orbitals, where $z$ denotes the direction perpendicular to the molecular plane. The transfer energies calculated from $\pi-\pi$ interaction change from the negative values of the side-by-side arrangement (relative angle $\theta=0$ ) to the positive values of the face-toface arrangement $\left(\theta=90^{\circ}\right)$, consistent with the transfer energy values listed in Table 1 . Using quantum chemical calculations, Mori et al. have shown that the transfer energy becomes zero around $\theta=10^{\circ}$ and the magnitude of the inter-column transfer energy is sensitive to small variations in the molecular arrangements [28]. In fact, we find that $t_{\mathrm{I} 1}$ and $t_{\mathrm{I} 2}$ are very sensitive to the anion replacement and the increase in the $t_{\mathrm{I} 1}$ and $t_{\mathrm{I} 2}$ magnitudes from the $\mathrm{PF}_{6}$ salt to the $\mathrm{ClO}_{4}$ salt is explained by the decrease of $\gamma$.

In the multi-transfer model, the energy band is described by

$$
\begin{aligned}
\epsilon(\mathbf{k})=\quad & \hbar v_{\mathrm{F}}\left(\left|k_{x}\right|-k_{\mathrm{F}}\right)-2 t_{b} \cos \left(b k_{y} \pm \phi\right) \\
& -\left(2 \tau_{\cos }-\epsilon_{0}\right) \cos 2\left(b k_{y} \pm \phi\right) \\
& \pm \tau_{\sin } \sin 2\left(b k_{y} \pm \phi\right)
\end{aligned}
$$

where $\tau_{\text {cos }}$ and $\tau_{\text {sin }}$ are the second harmonic coefficients of the energy dispersion expanded in trigonometric functions and $\phi$ is the phase shift of FS from the rectangular lattice model [26]. $\tau_{\cos }$ and $\tau_{\text {sin }}$ are given in terms of the inter-column transfer energies and are sensitive to small variations in the molecular arrangements. The angle $\phi$ plays a role only in the incommensurability of the SDW; however, it is not important for the goodness of the nesting, i.e., the stability of the SDW phase. If we ignore the second harmonic terms of Eq. (2), the FS can be nested perfectly with nesting vector $Q^{0}$ with the $k_{y}=(2 \phi-\pi) / b$. When the $\cos 2\left(b k_{y} \pm \phi\right)$ term is considered, the slightly distorted FS cannot be nested perfectly by $Q^{0}$, moreover, if the $t_{b}^{\prime}=\left|2 \tau_{\cos }-\epsilon_{0}\right|$ coefficient is large, the FISDW phase with $Q^{0}$ is suppressed. However, the $\sin 2\left(b k_{y} \pm \phi\right)$ term does not promote the suppression of the FISDW phase with $Q^{0}$. In contrast, the FISDW with $Q_{ \pm}$, in which $k_{y}=(4 \phi-\pi) / 2 b$ is not suppressed by the $\cos 2\left(b k_{y} \pm \phi\right)$ term but rather only by the $\sin 2\left(b k_{y} \pm \phi\right)$ term.

The coefficients of the dispersion relations $\left(\epsilon_{0}, \tau_{\cos }, \tau_{\sin }\right.$ and $t_{b}^{\prime}$ ) are presented in Table 1 . The $t_{b}^{\prime}=4.17 \mathrm{meV}$ in the $\mathrm{ClO}_{4}$ slat at ambient pressure is much larger than the $t_{b}^{\prime}=2.53 \mathrm{meV}$ in the $\mathrm{PF}_{6}$ salt at $0.7 \mathrm{GPa}$. Therefore, the FISDW phase with $Q^{0}$ is suppressed under pressure in the $\mathrm{ClO}_{4}$ salt but not in the $\mathrm{PF}_{6}$ salt. In contrast, the $\left|\tau_{\text {sin }}\right|=0.55 \mathrm{meV}$ in the $\mathrm{ClO}_{4}$ salt is much smaller than the $\left|\tau_{\text {sin }}\right|=0.92 \mathrm{meV}$ in the $\mathrm{PF}_{6}$ salt at $0.7 \mathrm{GPa}$. This result suggests that the FISDW phase with $Q_{ \pm}$is stabilized in the $\mathrm{ClO}_{4}$ salt but not in the $\mathrm{PF}_{6}$ salt at $0.7 \mathrm{GPa}$. Because the SDW ground states in the $\mathrm{PF}_{6}$ and $\mathrm{ReO}_{4}$ salts are suppressed by an almost identical hydrostatic pressures of $\sim 1.0 \mathrm{GPa}$, the chemical pressure and the coefficients of the dispersion relation of the two salts can be considered to be the same. This indicates that the $Q_{ \pm}$state of the FISDW phases in the $\mathrm{ReO}_{4}$ salt is suppressed by the large $\left|\tau_{\text {sin }}\right|$ while the $Q^{\mathrm{p}, \mathrm{n}}$ states are preserved because of the small value of $t_{b}^{\prime}$. These results lead to the conclusion that the difference of the FISDW phase between the $\mathrm{ReO}_{4}$ and $\mathrm{ClO}_{4}$ salts are caused by the different types of nesting vector $\left(Q^{0}\right.$ and $\left.Q_{ \pm}\right)$, determined by the difference of the chemical and hydrostatic pressures.

Conclusion. - We conducted Hall resistance and MR measurements under hydrostatic pressure and under magnetic field in the FISDW phase of the $\mathrm{ReO}_{4}$ salt. The quantum numbers of the quantized Hall resistance are determined as $N=0,1,2,-2,-4,-6,-8, \cdots$ with decreasing field. This series of $N$ containing the negative even- $N$ phase and the positive integer phase can be successfully explained by the "extended standard model" that considers the large AO gap. Moreover, we found that the difference in the $N$ series of the $\mathrm{ReO}_{4}$ and $\mathrm{ClO}_{4}$ salts can be explained by the difference of the molecular arrangements in the $a-b$ plane, which was determined by the hydrostatic and chemical pressures.

Acknowledgements. - We would like to thank the OPEN FACILITY of Hokkaido University for allowing us to use the Multi-Extreme conditions high field magnetism equipment for the Hall and magneto resistance measurements. 


\section{REFERENCES}

[1] Ishiguro T., Yamaji K. and Saito G., Organic Superconductors II (Springer-Verlag, Berlin) 1998.

[2] Pouget J. P., Shirane G., Bechgandd K. and Fabre J. M., Phys. Rev. B, 27 (1983) 5203.

[3] Osada T., Kagoshima S. and Miura N., Phys. Rev. Lett., 69 (1992) 1117

[4] Lebed A. G. and Bak P., Phys. Rev. B, 40 (1989) 11433

[5] McKernan S. K., Hannahs S. T., Scheven U. M., Danner G. M. and Chaikin P. M., Phys. Rev. Lett., 75 (1995) 1630

[6] Cho H. and Kang W., Phys. Rev. B, 59 (1999) 9814

[7] Zanchi D. and Montambaux G., Phys. Rev. Lett., 77 (1996) 366

[8] Dupuis N. and Yakovenko V. M., Phys. Rev. Lett., 80 (1998) 3618

[9] M. Ribault, Mol. Cryst. Liq. Cryst., 119 (1985) 91

[10] Scheven U. M., Chashechinana E. I., Lee E. and Chaikin P. M., Phys. Rev. B, 52 (1995) 3484

[11] Moret R., Ravy S., Pouget J. P., Comes R. and Bechgaard K., Phys. Rev. Lett., 57 (1986) 1915

[12] Colin C., Auban-Senzier P., Pasquier C. R. and Bechgaard K., Eur. Phys. Lett., 75 (2006) 301

[13] Kishigi K. and Machida K., Phys. Rev. B, 53 (1996) 5461

[14] Kang W., Cooper J. R. and Jérome D., Phys. Rev. B, 43 (1991) 11467

[15] Alemany P., Pouget J. P. and Canadell E., Phys. Rev. B, 89 (2014) 155124

[16] Uji S., Terashima T., Aoki H., Brooks J. S., Tokumoto M., Takasaki S., Yamada J. and Anzai H., Phys. Rev. B, 53 (1996) 14399

[17] Schwenk H., Parkin S. S. P., Schumaker R., Greene R. L. and Schweitzer D., Phys. Rev. Lett., 56 (1986) 667

[18] Nothardt A., Balthes E., Salameh B., Schweitzer D. and Sheikin I., J. Low Temp. Phys., 142 (2006) 485

[19] Stark R. W. and Friedberg C. B., J. Low Temp. Phys., 14 (1974) 111

[20] Although the estimated Hall resistance with $N=-2$ from the sample thickness is $R_{x y}(N=-2) \approx 0.024 \Omega$, it is approximately $30 \%$ smaller than the observed $R_{x y}$. It may be due to the slight inhomogeneity of the current density, which caused by the gold evaporated contacts.

[21] Hannahs S. T, Brooks J. S., Kang W., Chiang L. Y. and Chaikin P. M., Phys, Rev. Lett., 63 (1989) 1988

[22] T. Komada et al. Private communication.

[23] Matsunaga N., Briggs A., Ishikawa A., Nomura K., Hanajiri T., Yamada J., Nakatsuji S. and Anzai H., Phys. Rev. B, 62 (2000) 8611

[24] Matsunaga N., Ayari A., Monceau P., Ishikawa A., Nomura K., Watanabe M., Yamada J. and Nakatsuji S., Phys. Rev. B, 66 (2002) 024425

[25] Miyazaki M., Keita K. and Hasegawa Y., J. Phys. Soc. Jpn., 68 (1999) 313

[26] Yamaji K., J. Phys. Soc. Jpn., 55 (1986) 860

[27] Gallois B., Gaultier J., Hauw C., Chasseau D., Meresse A., Filhol A. and Bechgaard K., Mol. Cryst. Liq. Cryst., 119 (1985) 225 Gallois B., Gaultier J., Bechtel F., Filhol A. and Vettier C., Mol. Cryst. Liq. Cryst., 148 (1987) 279 Gallois B., Chasseau D.,
Gaultier J., Hauw C., Filhol A. and Bechgaard K., J. Phys. (Paris), 44 (1983) 1071

[28] Mori T., Kobayashi A., Sasaki Y., Kobayashi H., Saito G. and Inokuchi H., Bull. Chem. Soc. Jpn., $\mathbf{5 7}$ (1984) 627

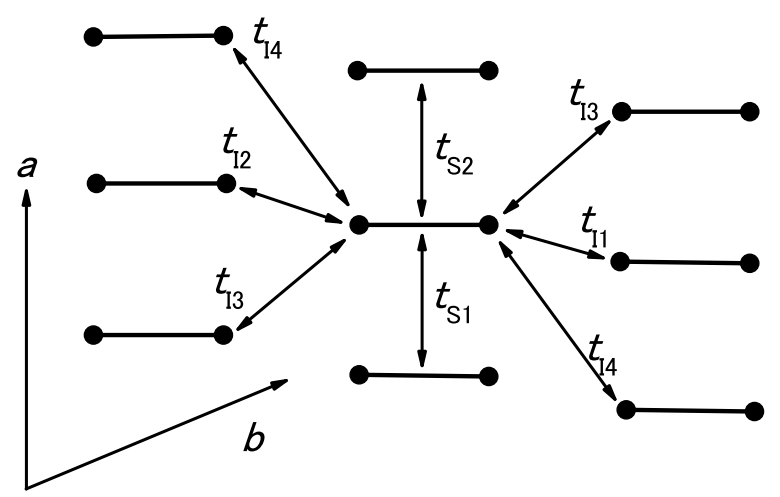

Fig. 4: Enlarged view of the inset figure of the Table 1. 